\title{
MicroRNA-195 protection against focal cerebral ischemia by targeting CX3CR1
}

\author{
*Guang Yang, MD, PhD, 1,2 Zhendong Liu, MD, ${ }^{1,2}$ Lu Wang, MD, PhD, ${ }^{3}$ Xin Chen, MD, PhD, \\ Xiaoxiong Wang, MD, ${ }^{1,2}$ Qi Dong, MD, PhD, ${ }^{4}$ Daming Zhang, MD, PhD, ${ }^{1,2}$ Zhao Yang, MD, ${ }^{1,2}$ \\ Qi Zhou, MD, ${ }^{5}$ Jingxian Sun, MD, ${ }^{1,2}$ Linmeng Xue, MD, ${ }^{1,2}$ Xinzhuang Wang, MD, ${ }^{1,2}$ Ming Gao, MD, ${ }^{1,2}$ \\ Lili Li, BS, ${ }^{1}$ Ran Yi, MD, PhD, ${ }^{6}$ Gareev Ilgiz, MD, ${ }^{1,7}$ Jing Ai, MD, PhD, ${ }^{8}$ and \\ Shiguang Zhao, MD, PhD ${ }^{1,2}$
}

${ }^{1}$ Department of Neurosurgery, The First Affiliated Hospital of Harbin Medical University, Harbin; ${ }^{2}$ nnstitute of Brain Science, Harbin Medical University, Harbin; ${ }^{2}$ Department of Urology, The Fourth Hospital of Harbin Medical University, Harbin; ${ }^{4}$ Department of Neurology, The First Affiliated Hospital of Harbin Medical University, Harbin; ${ }^{5}$ Research Administration Office, The First Affiliated Hospital of Harbin Medical University, Harbin; ${ }^{6}$ Department of Endocrinology and Metabolism, The First Affiliated Hospital of Harbin Medical University, Harbin, China; 'Department of Medical Rehabilitation with courses of Neurosurgery and Acupuncture IAPE, Bashkir State Medical University, Ufa, Republic of Bashkortostan, Russia; and ${ }^{8}$ Department of Pharmacology, The State-Province Key Laboratories of Biomedicine-Pharmaceutics of China, College of Pharmacy of Harbin Medical University, Harbin, China

OBJECTIVE It has been reported that microRNA-195 (miR-195) protects against chronic brain injury induced by chronic brain hypoperfusion. However, neither the expression profile of miR-195 nor its potential role during acute ischemic stroke has been investigated. In this study, the authors' aim was to verify the mechanism of miR-195 in acute ischemic stroke.

METHODS The plasma levels of miR-195 expression were assessed using real-time PCR in 96 patients with acute ischemic stroke, and the correlation with the National Institutes of Health Stroke Scale score was evaluated. In addition, cerebral infarct volume, neurological score, and levels of miR-195 and CX3CL1/CX3CR1 mRNA and protein expression were assessed in mice subjected to middle cerebral artery occlusion (MCAO) with or without intra-cerebroventricular infusion of lentiviral vector. The inflammatory cytokines tumor necrosis factor $-\alpha$ (TNF $\alpha$ ), interleukin (IL) $-1 \beta$, and IL- 6 of mouse brains after MCAO and BV2 cells treated with oxygen-glucose deprivation were measured using enzyme-linked immunosorbent assay, and apoptotic proteins were examined by Western blotting. Direct targeting of CX3CL1/CX3CR1 by miR-195 was determined by immunoblotting and dual luciferase assay.

RESULTS In ischemic stroke patients, miR-195 was significantly downregulated and expression levels of miR-195 in these patients negatively correlated with the National Institutes of Health Stroke Scale score. In mice after MCAO, miR195 overexpression decreased infarct volume, alleviated neurological deficits, and most importantly, suppressed an inflammatory response. Meanwhile, miR-195 suppressed the expression of the inflammatory cytokines TNF $\alpha$, IL-1 $\beta$, and IL-6 in vitro and in vivo. The authors further discovered that both CX3CL1 and CX3CR1 are direct targets of miR-195, but miR-195 exerts neuroprotective roles mainly through inhibiting CX3CR1-mediated neuroinflammation and subsequent neuronal cell apoptosis.

CONCLUSIONS Taken together, these findings suggest that miR-195 promotes neuronal cell survival against chronic cerebral ischemic damage by inhibiting CX3CR1-mediated neuroinflammation. This indicates that miR-195 may represent a novel target that regulates neuroinflammation and brain injury, thus offering a new treatment strategy for cerebral ischemic disorders.

https://thejns.org/doi/abs/10.3171/2018.5.JNS173061

KEYWORDS CX3CL1; CX3CR1; ischemic stroke; microRNA-195; neuroinflammation; vascular disorders

ABBREVIATIONS ELISA = enzyme-linked immunosorbent assay; GAPDH = glyceraldehyde 3-phosphate dehydrogenase; $I L=$ interleukin; $L v=$ lentivirus; $M C A O=$ middle cerebral artery occlusion; miR-195 = miRNA-195; miRNA = microRNA; NIHSS = National Institutes of Health Stroke Scale; ODN = oligodeoxynucleotide; OGD = oxygenglucose deprivation; qRT-PCR = quantitative real-time polymerase chain reaction; TNF $\alpha=$ tumor necrosis factor- $\alpha ;$ TTC $=2,3,5$-triphenyltetrazolium chloride; UTR = untranslated region.

SUBMITTED January 21, 2018. ACCEPTED May 29, 2018.

INCLUDE WHEN CITING Published online November 23, 2018; DOI: 10.3171/2018.5.JNS173061.

${ }^{*}$ G.Y., Z.L., L.W., and X.C. contributed equally to this work. 
I SCHEMIC stroke is known to be a leading cause of death and long-term disability. ${ }^{12,23}$ It is caused by an insufficient blood supply to the brain and is characterized by hypoxia, excitotoxicity, and inflammation, eventually leading to neuronal cell death. ${ }^{17}$ Substantial research has been performed to uncover the pathophysiological mechanisms underlying ischemic brain injury and to identify molecular targets for stroke. ${ }^{18,22}$ To date, clinical therapy has improved somewhat in the acute ischemic period; however, long-term therapies remain limited. ${ }^{4,9}$ Therefore, further exploration of the molecular mechanisms responsible for cerebral ischemia-induced brain injury is of great importance for developing effective therapeutic targets for acute ischemic stroke.

A myriad of complex signaling molecules and pathways involved in ischemic stroke have been identified. Among these, microRNAs (miRNAs) have recently attracted attention for their pivotal regulatory function in gene expression. Emerging studies have shown that miRNAs might be the pivotal mediators of ischemic pathological changes and may serve as therapeutic targets and prognostic markers in stroke. ${ }^{11,25}$ However, the changes in and roles of miRNAs in response to ischemic injury are complex and should be examined in more detail through further investigation.

Of the known ischemia-sensitive miRNAs, we found that miRNA-195 (miR-195) is downregulated in mice with chronic brain hypoperfusion induced by bilateral common carotid artery occlusion. ${ }^{1,2,14,20}$ Therefore, miRNA-195 may serve a protective role in chronic cerebral ischemia-induced brain injury. However, so far, neither the change in expression of miR-195 nor its potential role in acute ischemic stroke has been explored. It is known that an individual miRNA can regulate the expression of numerous genes. In addition to the reported target genes of miR-195, ${ }^{24}$ through computational analysis, we determined that CX3CL1 and CX3CR1 may be novel targets of miR-195. The CX3CL1/ CX3CR1 signaling pathway has been shown to play a key regulatory role in the neuroinflammatory response..$^{16,21}$ An inflammatory response is generally found accompanying ischemic stroke and is believed to contribute to neuronal injury. Accordingly, based on the protective role of miR195 in chronic brain injury, as well as its predicted target of the CX3CL1/CX3CR1 pathway, we hypothesized that miR-195 likely exerts its neuroprotective effects against acute cerebral ischemic insults by inhibiting the CX3CL1/ CX3CR1-mediated inflammatory response and subsequently suppressing neuronal cell death.

\section{Methods}

\section{Clinical Data and Blood Collection}

Clinical data and blood samples were collected from 96 ischemic stroke patients who underwent treatment in the Department of Neurology at the First Affiliated Hospital of Harbin Medical University in China between January 2015 and December 2015. The diagnosis of ischemic stroke was based on clinical symptoms, neurological examination, and results of CT or MRI of the brain as defined according to the World Health Organization criteria. Clinical severity was assessed at admission using the
National Institutes of Health Stroke Scale (NIHSS) score. The baseline characteristics of the enrolled stroke patients are shown in Table 1. Forty-five nonstroke patients who experienced trauma and had diseases of other systems but were neurologically normal and had no medical history of stroke were recruited into this study as controls. For all stroke patients, blood samples were drawn within 72 hours from stroke onset. The study was approved by the institutional review board of Harbin Medical University, and the participants gave informed consent.

\section{Animals and In Vivo Gene Transfer}

All experimental protocols involving animals used in this study were carried out in accordance with the Guide for Animal Experimentation of Harbin Medical University and were approved by the Institutional Animal Care and Use Committee at Harbin Medical University. Male C57BL/6 mice, 8 to 10 weeks old $(n=270$ total), were randomly subjected to lentivirus-mediated gene transfer and a subsequent focal brain ischemia/reperfusion surgical procedure as described below. ${ }^{19}$ The mice were anesthetized with $2 \%$ isoflurane through a facemask and placed in a stereotactic frame. A 26-gauge brain infusion cannula was placed stereotactically into the left lateral ventricle (bregma, $-0.58 \mathrm{~mm}$; dorsoventral, $2.1 \mathrm{~mm}$; lateral, 1.2 $\mathrm{mm})$ as previously described. A 2- $\mu$ l solution of lentivirus (GeneCopoeia) was injected into the brain according to the manufacturer's instructions. Cerebral ischemia and reperfusion were performed 48 hours later.

\section{Model of Focal Cerebral Ischemia}

The mice were anesthetized with $2 \%$ isoflurane through a facemask. Focal cerebral ischemia and reperfusion was induced by middle cerebral artery occlusion (MCAO) lasting 1 hour with a silicone-coated 6-0 monofilament and followed by reperfusion, as previously described. ${ }^{19}$ Shamoperated mice underwent an identical procedure without inserting the suture. Rectal temperature was maintained at $37^{\circ} \mathrm{C} \pm 0.5^{\circ} \mathrm{C}$ with a heating pad. Mice with no evidence of acute neurological deficits after ischemia, those that died before 24 hours, and those with evidence of hemorrhage at the time of death were excluded from analysis. No significant difference $(\mathrm{p}<0.05)$ was observed between treatment groups in terms of the number of excluded animals.

\section{Tissue Preparation}

Mice were euthanized by cervical dislocation, and the brains were quickly removed, frozen in liquid nitrogen, and stored at $-80^{\circ} \mathrm{C}$ until processing and used for Western blot, quantitative real-time polymerase chain reaction (qRT-PCR), and enzyme-linked immunosorbent assay (ELISA) analysis. Blood samples were collected in EDTA-coated tubes, spun for 10 minutes at $500 \mathrm{~g}$ at $4{ }^{\circ} \mathrm{C}$, and stored at $-80^{\circ} \mathrm{C}$ until further processing.

\section{Assessment of Neurological Scores and Infarct Volumes}

Neurological deficit scores 24 hours after ischemia were evaluated by using a scoring system that ranged from 0 to 4 as follows: 0 , no deficit; 1 , failure to extend right forepaw; 2 , circling to the contralateral side; 3 , falling to 
TABLE 1. Baseline characteristics of patients with ischemic stroke

\begin{tabular}{lc}
\hline \multicolumn{1}{c}{ Characteristic } & Value \\
\hline No. of patients & 96 \\
\hline Median age, $\mathrm{yrs}(\mathrm{IQR})$ & $61.5(53-71)$ \\
\hline Male sex, $\mathrm{n}(\%)$ & $60(62.5)$ \\
\hline Hypertension, $\mathrm{n}(\%)$ & $52(54.16)$ \\
\hline Diabetes mellitus, $\mathrm{n}(\%)$ & $26(27.08)$ \\
\hline Cigarette smoking, $\mathrm{n}(\%)$ & $29(30.21)$ \\
\hline Alcohol drinking, $\mathrm{n}(\%)$ & $18(18.79)$ \\
\hline Median admission NIHSS score (IQR) & $6(2-11)$ \\
\hline Median time from onset to inclusion, hrs (IQR) & $19.25(6.38-36)$ \\
\hline
\end{tabular}

the right; and 4, not walking spontaneously, depressed level of consciousness.

After assessment of the neurological score, the mice were anesthetized and decapitated, and the brains were removed. The brains were sliced into 2-mm-thick sections using a rodent brain matrix, and staining was performed using 2,3,5-triphenyltetrazolium chloride (TTC; SigmaAldrich). The slices were stained with $2 \%$ TTC at $37^{\circ} \mathrm{C}$ for 30 minutes, fixed in $4 \%$ paraformaldehyde, and photographed. Infarct volume with edema correction as a percentage of the contralateral hemisphere was determined as follows: $100 \% \times$ (contralateral hemisphere volume - noninfarct ipsilateral hemisphere volume)/contralateral hemisphere volume.

\section{Cell Culture, Transfection, and Oxygen-Glucose Deprivation}

BV2 microglial cells and HEK293T cells were obtained from the National Infrastructure of Cell Line Resource. All cell lines were maintained in DMEM (Invitrogen) supplemented with 10\% fetal bovine serum (Invitrogen). MiR-195 mimics, scrambled control miRNA (GenePharma), or reporter plasmid was transfected into cultured cells using Lipofectamine 2000 reagent (Invitrogen) following the manufacturer's instructions. Twenty-four hours after transfection of miR-195 mimics or negative control, oxygen-glucose deprivation (OGD) was performed as described previously. ${ }^{10}$ Briefly, BV2 cells were exposed to glucose-free DMEM and cultured in an incubator in an atmosphere of $1 \% \mathrm{O}_{2}, 5 \% \mathrm{CO}_{2}$, and $94 \% \mathrm{~N}_{2}$ for 3 hours at $37^{\circ} \mathrm{C}$.

\section{TUNEL Staining}

The brain tissues were cut into $20-\mu \mathrm{m}$ frozen coronal sections and cell death was detected using the In Situ Cell Death Detection Kit-POD (Roche) following the manufacturer's instructions. The nuclei were stained with DAPI. Sections were observed under a fluorescence microscope (Carl Zeiss). The percentage of TUNEL-positive nuclei was used as an apoptotic index.

\section{ELISA}

The expression of the proinflammatory cytokines interleukin (IL) $-1 \beta$, IL- 6 , and tumor necrosis factor- $\alpha$ $(\mathrm{TNF} \alpha)$ in brain homogenates and in the culture medium of BV2 cells was determined using commercially available ELISA kits according to the manufacturer's instructions (R\&D Systems).

\section{Masking Antisense Oligodeoxynucleotides}

The CX3CL1-masking antisense oligodeoxynucleotides (CX3CL1-ODNs; GGUUUUGAGAGGAGACGAC GAC) and CX3CR1-masking antisense ODNs (CX3CR1ODNs; CUUCUCGGACGUCCACGACGAC), which had 5 nucleotides or deoxynucleotides at both ends of the antisense molecules, were locked by a methylene bridge connecting the 2'-O and the 4'-C atoms and masked the binding sites of miR-195 located in position 228-252 of the CX3CL1 3' untranslated region (UTR) or position 12201244 of the CX3CR1 3'UTR, which were synthesized by Sangon Biological Engineering Technology and Service.

\section{Dual Luciferase Reporter Assay}

HEK293T cells were transfected with miR-195 mimics or control miRNA as well as psi-CHECK-2-target DNA (firefly luciferase vector) and a blank plasmid using Lipofectamine 2000 transfection reagent (Invitrogen). Luciferase activity was measured with a dual luciferase reporter assay kit and luminometer (both Promega) 48 hours after transfection. Nucleotide-substitution mutagenesis was performed using direct oligomer synthesis for the 3'UTRs of CX3CL1 or CX3CR1. All constructs were sequence verified.

\section{RNA Extraction and qRT-PCR}

Total RNA was isolated from specimens and cells using a Trizol standard protocol (Invitrogen) and extracted from plasma using the miRNeasy Serum/Plasma Kit (QIAGEN). Quantitative RT-PCR was performed in triplicate using the ABI 7900 fast real-time PCR System (Applied Biosystems) and normalized with U6 and glyceraldehyde 3-phosphate dehydrogenase (GAPDH) endogenous control. MiR-195 and U6 levels were measured using the TaqMan microRNA assay kit, and mRNA levels of CX3CL1, CX3CR1, and GAPDH were detected using the SYBR Green PCR Master Mix kit in accordance with the manufacturer's instructions (Applied Biosystems).

\section{Protein Extraction and Western Blot}

Total proteins were extracted from specimens and cells using radioimmunoprecipitation assay buffer to lyse the cells and tissues. Lysate was separated by $10 \%$ sodium dodecyl sulfate-polyacrylamide gel electrophoresis, and the gel was blotted onto polyvinylidene membrane (Millipore). The membrane was blocked in 5\% nonfat milk and then incubated with rabbit anti-human CX3CL1 (1:1000, Abcam), CX3CR1 (1:500, Abcam), caspase-3 (1:1000, Cell Signaling Technology), or mouse anti-human GAPDH (1:1000, Santa Cruz). After washing, the membrane was incubated with fluorescence-conjugated anti-rabbit or anti-mouse $\operatorname{IgG}(1: 4000$, Invitrogen). The bound secondary antibody was quantified using Odyssey software (version 1.2 , LI-COR) by measuring the band intensity (area $\times$ optical density) for each group and normalized with GAPDH. 

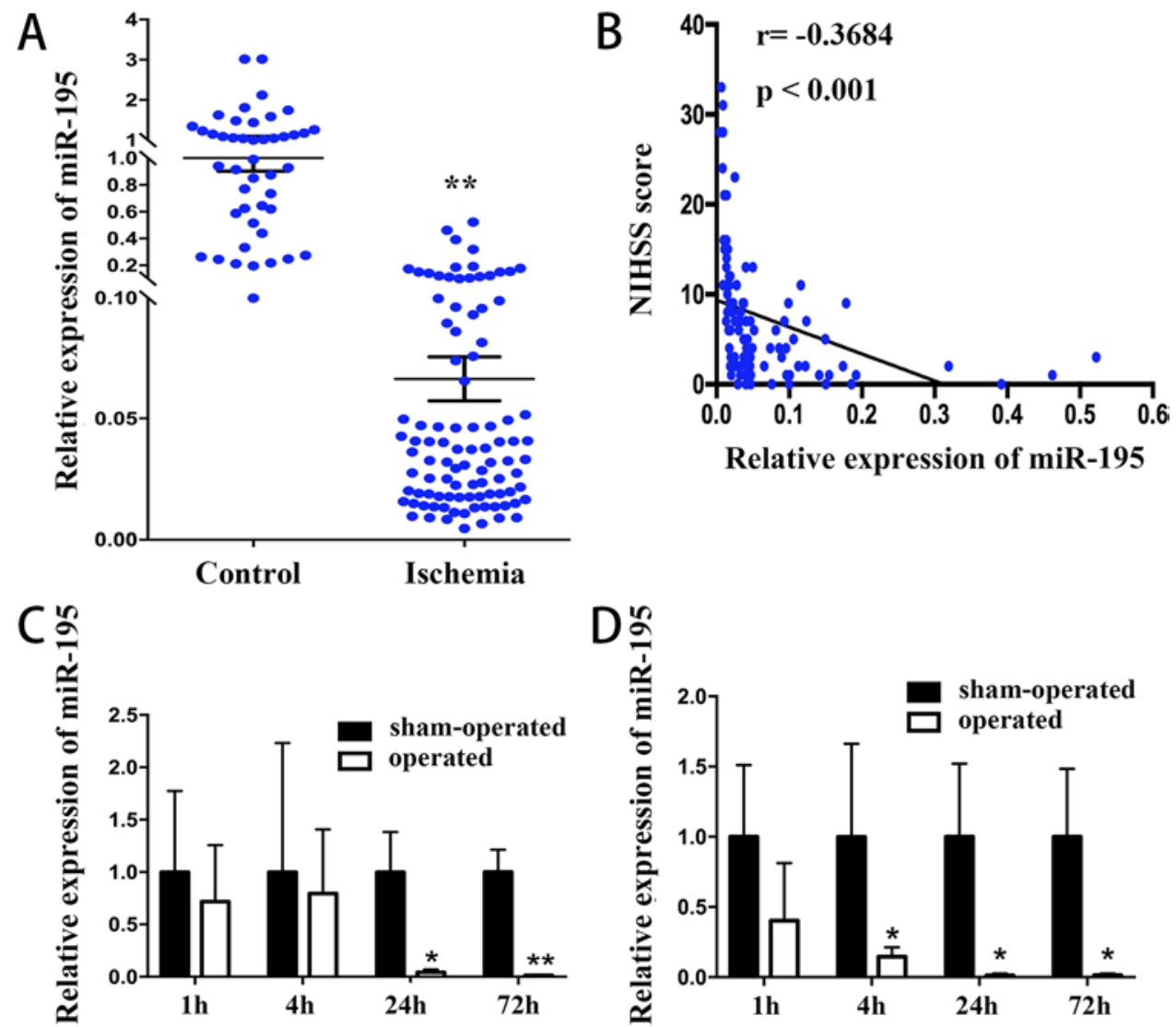

FIG. 1. Low expression of miR-195 in ischemic stroke. A: The expression levels of miR-195 in plasma in patients with acute ischemic stroke as assessed by qRT-PCR. B: Correlation between miR-195 expression level and NIHSS score in patients. C and D: The expression levels of miR-195 in the periinfarct cortex (C) and blood (D) of mice by qRT-PCR at 1, 4, 24, and 72 hours after ischemia. Data are presented as the mean \pm SEM for 3 separate experiments performed in duplicate. ${ }^{*} p<0.05 ;{ }^{* *} p<0.001$. Figure is available in color online only.

\section{Statistical Analysis}

Statistical analysis was performed using SPSS software (version 13.0, SPSS Inc.). The Student t-test, ANOVA, chisquare analysis, or Mann-Whitney test was applied, where appropriate; $p<0.05$ was considered statistically significant.

\section{Results \\ Low Expression of Plasma miR-195 in Ischemic Stroke Patients}

To verify the expression profiles of miR-195 in ischemic stroke patients, we detected the plasma levels of miR-195 expression in 96 patients with acute ischemic stroke using qRT-PCR. Our data showed that miR-195 levels were significantly lower in acute ischemic stroke patients than in the nonstroke patients (Fig. 1A). Furthermore, in order to explore the correlation between the miR-195 levels and severity of ischemic stroke, we compared the miR-195 expression level with the NIHSS score for each patient. A significant negative correlation was found between miR195 expression level and NIHSS score (Fig. 1B). Our results indicated that decreased miR-195 levels might play a role in ischemic stroke.

\section{MiR-195 Expression Is Downregulated in a Mouse Model of Cerebral Ischemia}

To assess the effects of miR-195 on cerebral ischemia reperfusion injury, we detected the expression levels of miR-195 in the periinfarct cortex and blood of mice by qRT-PCR at 1, 4, 24, and 72 hours after ischemia. Our data showed that miR-195 levels were significantly decreased at 24 hours after ischemia and were even found at low levels at 72 hours after ischemia relative to sham-operated mice (Fig. 1C and D). These findings were similar to those of ischemic stroke patients and further suggest that miR195 may be involved in the underlying mechanisms after cerebral ischemia reperfusion.

\section{MiR-195 Protects the Brain From Transient Focal Cerebral Ischemia}

To further explore the potential biological effects of miR-195 in cerebral ischemia reperfusion, we performed intraventricular infusions of lentivirus (Lv)-miR-195 or Lv-scrambled control. After MCAO, mice infused with Lv-miR-195 showed a significant increase in miR-195 expression compared with mice infected with Lv-scrambled 24 hours after ischemia (Fig. 2A). After Lv-miR-195 infusion, the MCAO infarct volume was significantly de- 

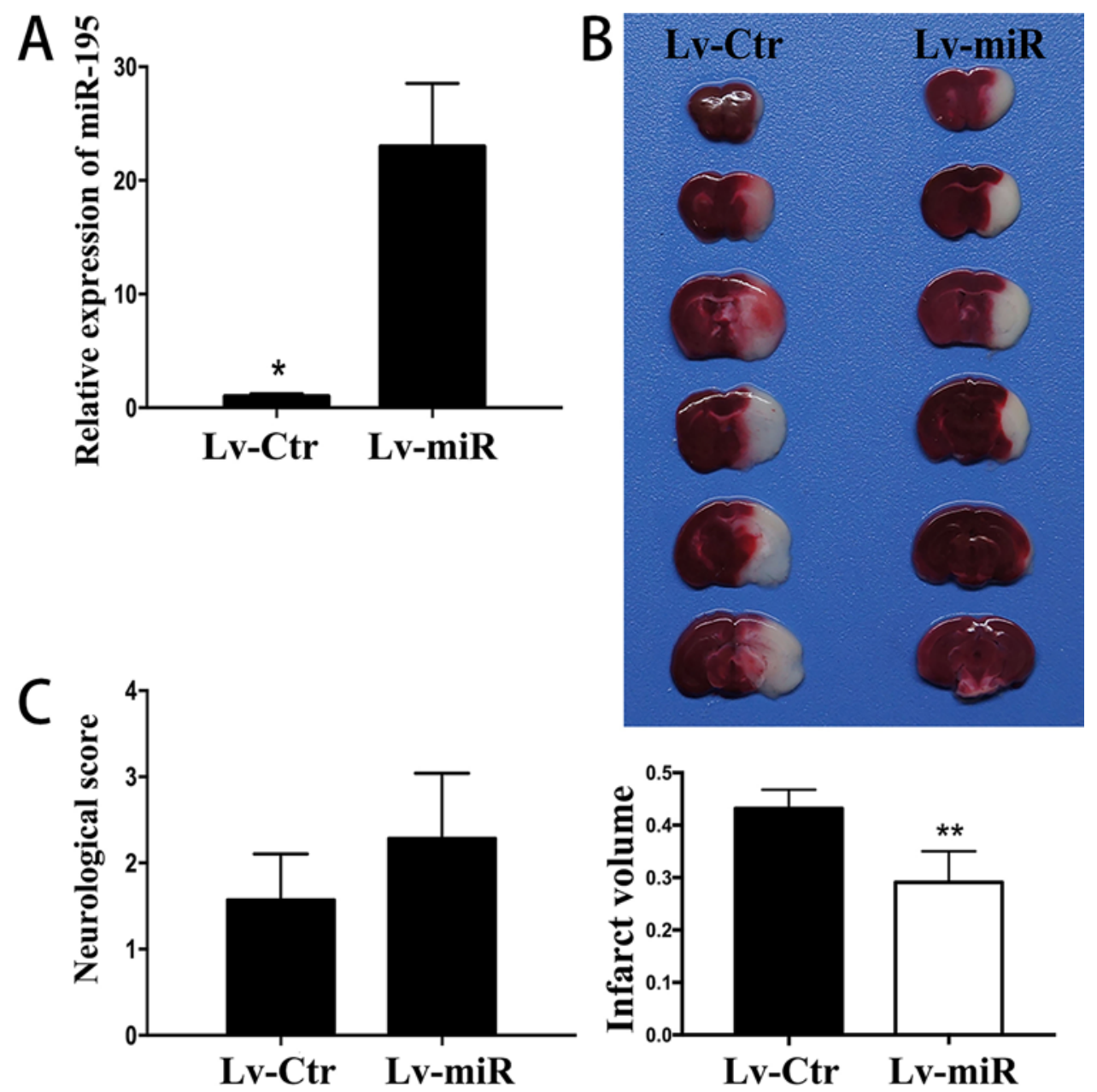

FIG. 2. MiR-195 protects the brain from focal cerebral ischemia. A: miR-195 expression was quantified by qRT-PCR in the mouse brain with cerebral ischemia reperfusion after infusions of Lv-miR-195 (Lv-miR) or Lv-scrambled control (Lv-Ctr). B: Cerebral infarct volume was evaluated using TTC staining of coronal brain sections. Quantitative analysis was performed to measure infarct volume. C: The neurological score was estimated in mice infused with Lv-miR-195 or Lv-control after MCAO. Data are presented as the mean \pm SEM for 3 separate experiments performed in duplicate. ${ }^{*} p<0.05$; ${ }^{* *} p<0.001$. Figure is available in color online only.

creased (Fig. 2B). Gross motor function, estimated by neurological score 24 hours after ischemia, was improved following Lv-miR-195 infusion (Fig. 2C). These results suggest that miR-195 protects the brain from transient focal cerebral ischemia.

\section{MiR-195 Suppresses Neuronal Cell Apoptosis}

To determine the relevant mechanisms of miR-195 expression in cerebral ischemia injury, we initially compared the apoptotic response between mice infused with Lv-miR-195 and those infused with Lv-scrambled. Western blot analysis showed that the cleaved caspase- 3 protein was markedly decreased in the periinfarct areas of the mouse brain infused with Lv-miR-195 relative to those with Lv-scrambled (Fig. 3A). Similarly, the number of TUNEL-positive nuclei was also significantly reduced in the periinfarct areas of the mouse brain infused with LvmiR-195 (Fig. 3B). These results suggest that suppressing neuronal cell apoptosis may be a mechanism involved in the neuroprotective effects of miR-195.

\section{MiR-195 Suppresses Inflammatory Signaling In Vitro and In Vivo}

To further investigate whether miR-195 is involved in inflammatory signaling of microglia under ischemia, we tested the effects of exogenous miR-195 on the expression of the microglia cytokines TNF $\alpha$, IL-1 $\beta$, and IL- 6 in vitro and in vivo. First, we explored the effects of miR-195 on the expression of TNF $\alpha$, IL-1 $\beta$, and IL- 6 in the brains of the ischemic mice. ELISA also showed that the levels of IL-6 as well as IL- $1 \beta$ and TNF $\alpha$ proteins in the periinfarct areas of mouse brains infected with Lv-miR-195 were significantly lower than those in the brains in the Lv-scrambled group (Fig. 3C). Subsequently, the BV2 microglial cells underwent OGD after infusion with miR-195 mimics or scrambled control for 24 hours. We found that the levels of TNF $\alpha$ and IL-1 $\beta$ in the cells treated with miR-195 mimics were significantly lower than the levels in the cells treated with scrambled control (Fig. 3D). These findings indicate that the increased expression of miR-195 could suppress inflammatory signaling after MCAO. 


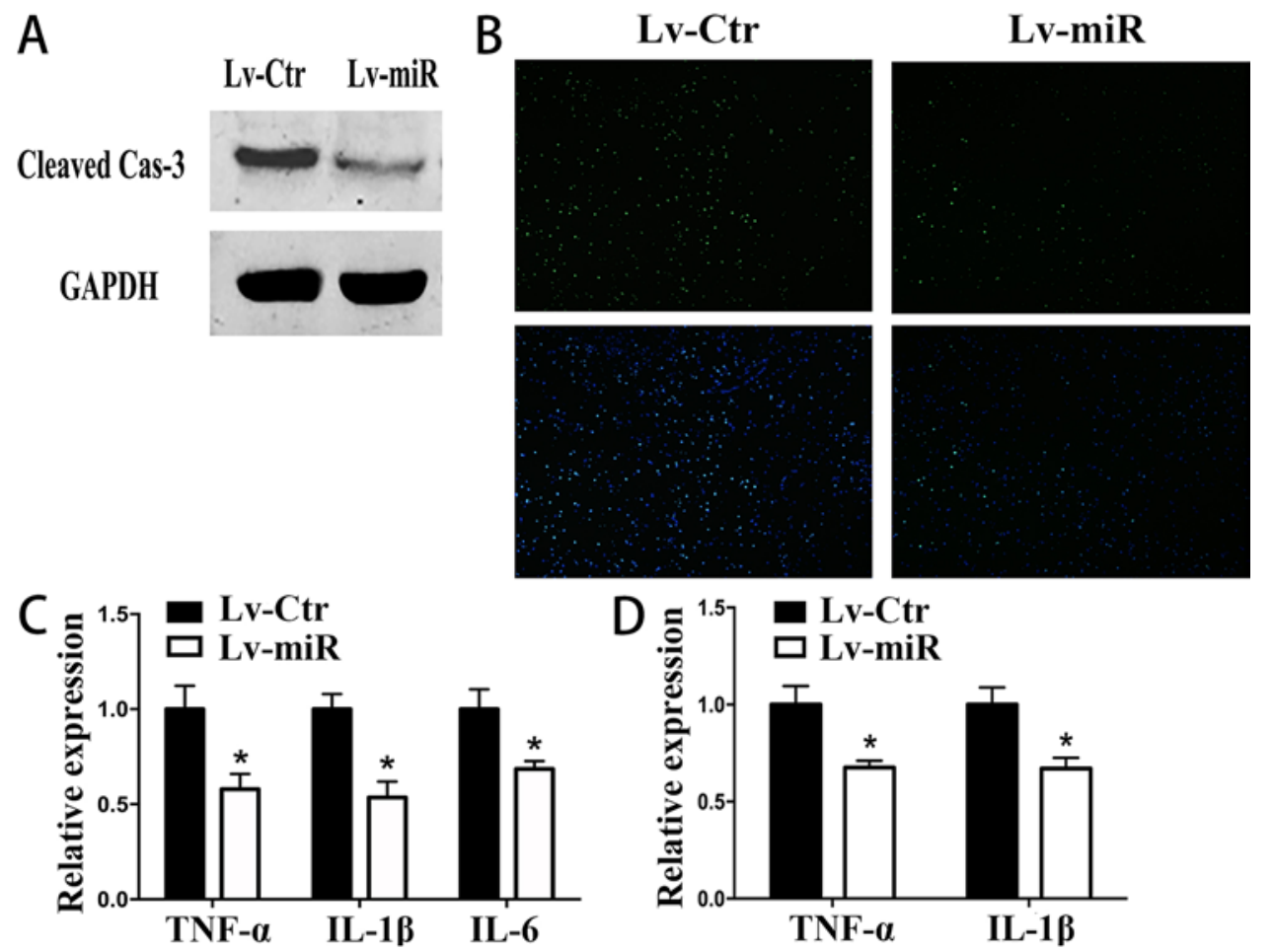

FIG. 3. MiR-195 and its neuroprotective effects. A: The caspase-3 protein was determined by Western blotting in post-MCAO mouse brains infected with LV-miR-195 relative to LV-scrambled control. B: The apoptotic cells are shown by TUNEL in the periinfarct areas of the mouse brain. C and D: Expression levels of TNF $\alpha$, IL-1 $\beta$, and IL- 6 were detected using ELISA in the periinfarct areas of the mouse brains (C) and the BV2 cells treated with OGD (D). Data are presented as the mean \pm SEM for 3 separate experiments performed in duplicate. ${ }^{*} p<0.05$. Figure is available in color online only.

\section{CX3CL1 and CX3CR1 Protein Levels Inversely Correlate With the miR-195 Level After MCAO}

The CX3CL1/CX3CR1 signaling pathway has been shown to play an important role in brain ischemic injury. More importantly, the databases (miRanda, PicTar, and TargetScan) predicted that CX3CL1 and CX3CR1 may be common target genes for miR-195 (Fig. 4A). We wondered whether CX3CR1 plays an important role together with CX3CL1 in the process of miR-195-mediated ischemia/ reperfusion injury. To test this hypothesis, we assessed the expression levels of CX3CL1 and CX3CR1 proteins in the periinfarct areas of mouse brains infected with LvmiR-195. We observed a significant decrease of CX3CL1 and CX3CR1 in Lv-miR-195-infected mice relative to Lv-scrambled controls (Fig. 4B). Taken together, our findings demonstrate that the miR-195 expression level in post-MCAO brain specimens was strongly correlated with CX3CL1 and CX3CR1 protein levels.

\section{CX3CL1 and CX3CR1 as Common Direct Targets of miR-195}

To further determine whether miR-195 regulates CX3CL1 and CX3CR1 at the protein and mRNA levels, Western blot and qRT-PCR analyses were performed in BV2 cells transfected with miR-195 mimics or scrambled control. Our Western blot results showed that expression of both CX3CL1 and CX3CR1 proteins in cells transfected with miR-195 mimics was downregulated compared with expression of these proteins in cells infected with scrambled control (Fig. 4C). However, qRT-PCR analysis revealed that the expression of CX3CL1 and CX3CR1 mRNA showed no significant differences in changes between cells transfected with miR-195 mimics and scrambled control (Fig. 4D). Moreover, CX3CL1 and CX3CR1 mRNA expression was also unchanged, but CX3CL1 and CX3CR1 protein expression was significantly decreased in mice infected with Lv-miR-195 (Fig. 4B and E). These data indicate that miR-195 can control CX3CL1 and CX3CR 1 by translational suppression rather than by mRNA degradation.

To assess whether there is a direct interaction between miR-195 and CX3CL1 or CX3CR1, wild-type or mutant 3'UTR of CX3CL1 and CX3CR1 genes was respectively incorporated into the dual luciferase reporter plasmid system (Fig. 4A). Subsequently, miR-195 mimic or scrambled control was cotransfected with the 3'UTR of CX3CL1 or CX3CR1 dual luciferase reporter plasmid into HEK293T cells. We observed that miR-195 decreased the relative luciferase activity of the reporter containing the wild-type 3'UTR of CX3CL1 and CX3CR1 (Fig. 4F and G). However, mutations in the predicted binding site of miR-195 in the 3'UTR of CX3CL1 or CX3CR1 genes abrogated the aforementioned inhibitory effect of miR-195, demonstrating that CX3CL1 and CX3CR1 are common direct targets of miR-195.

\section{Neuroprotective Effect of miR-195 Relies Mainly on Inhibiting CX3CR1}

To investigate the interactive role of CX3CL1 and 
A

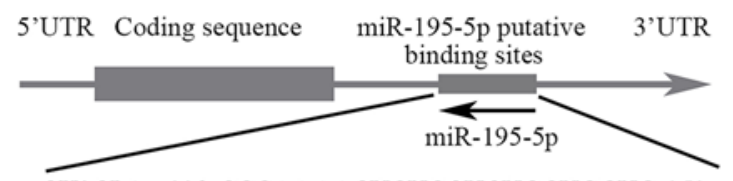

CX3CL1 228-CCCAAAACUCUCCUCUGCUGCUG-252

|| || || |

miR-195-5p 3' CGGUUAUAAAGACACGACGAU 5'

$\vdots \vdots \vdots \vdots \vdots \vdots \vdots \vdots$

CX3CL1 Mut 228-CCCAAAACUCUCCUCGAACTACG-252

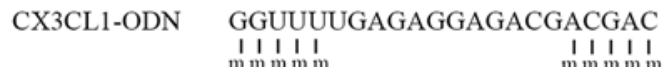

CX3CR1 1220-GGAAGAGCCUGCAGGUGCUGCUG-1244

|ा|ा|ा

miR-195-5p 3' CGGUUAUAAAGACACGACGAU 5'

: : : $: \vdots: ! \vdots$

CX3CR1 Mut 1220-GGAAGAGCCUGCAGGGAACTACG-1244

CX3CR1-ODN CUUCUCGGACGUCCACGACGAC

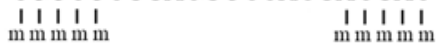
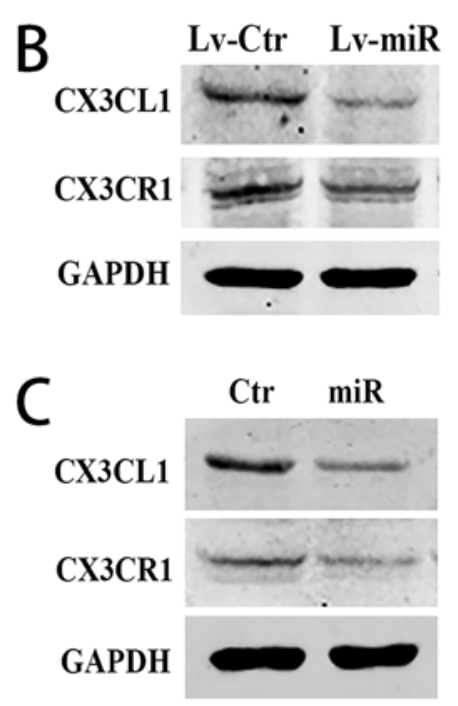
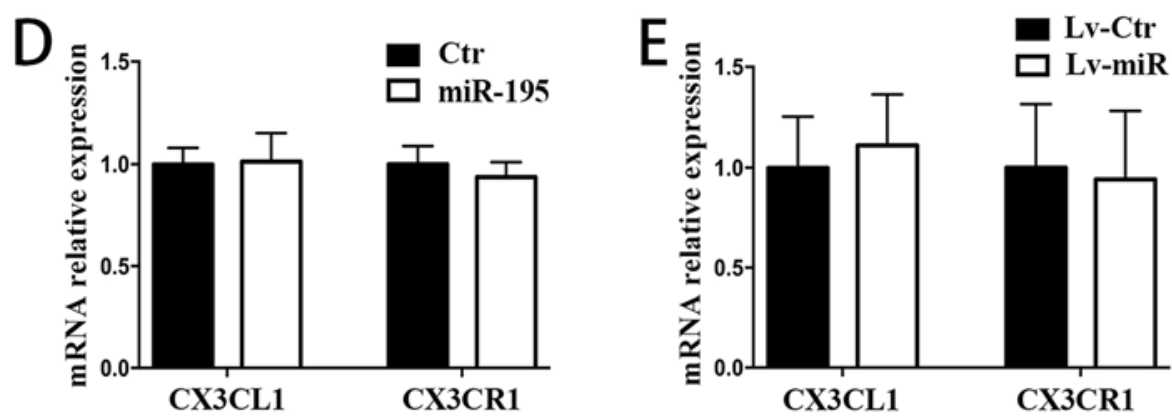

$\mathrm{F}$
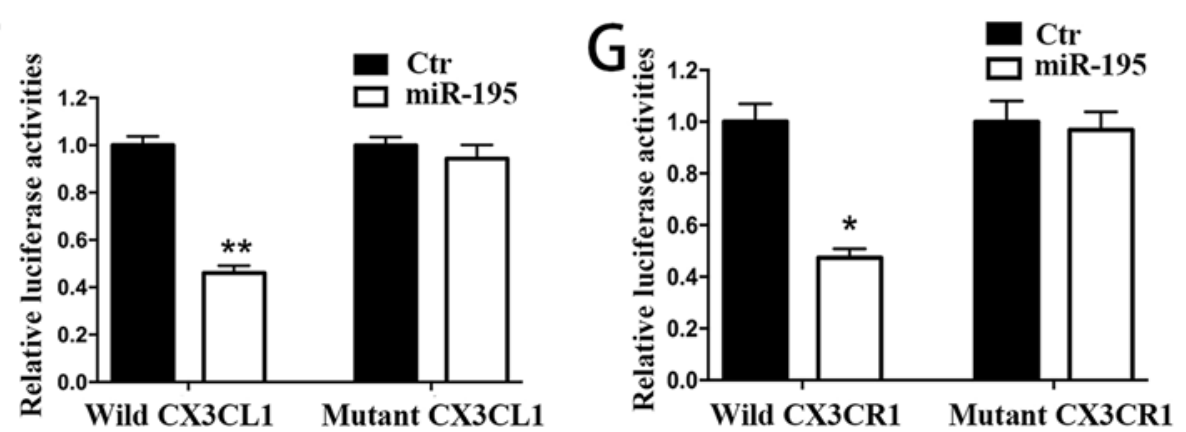

FIG. 4. CX3CL1 and CX3CR1 are direct targets of miR-195. A: The potential interaction between miR-195 and putative binding sites in the CX3CL1 and CX3CR1 $3^{\prime} U T R$, respectively. The mutant sequences are equivalent to the wild-type ones except mutations at the $3^{\prime}$ end of target site are underlined. The sequences of CX3CL1-ODN and CX3CR1-ODN are shown. $m=$ methylation. $B$ : CX3CL1 and CX3CR1 protein expression is determined by Western blotting in the periinfarct areas of mouse brains infected with LV-miR-195 or Lv-scrambled control. C: CX3CL1 and CX3CR1 protein expression was determined by Western blotting in the BV2 cells transfected with miR-195 mimics or scrambled control. D: CX3CL1 and CX3CR1 mRNA expression levels were determined by qRT-PCR in the BV2 cells transfected with miR-195 mimics or scrambled control. E: CX3CL1 and CX3CR1 mRNA expression levels were determined by qRT-PCR in the periinfarct areas of mouse brains infected with Lv-miR-195 or Lv-scrambled. F and G: Luciferase activities were analyzed in BV2 cells 48 hours after cotransfection of miR-195 mimics and either wild-type or mutant CX3CL1 and CX3CR1 $3^{\prime} U T R$. Data are presented as mean \pm SEM for 3 separate experiments performed in duplicate. ${ }^{*} p<0.05$. Figure is available in color online only.

CX3CR1 in the miR-195-involved neuroprotective effect, we constructed the CX3CL1 or CX3CR1 masking antisense ODNs as previously reported..$^{20}$ The sequences of CX3CL1-ODNs and CX3CR1-ODNs are shown in Fig. 4A, and the inhibitory effect of miR-195 on CX3CL1 or CX3CR1 expression was abolished by cotransfection with CX3CL1-ODN or CX3CR1-ODN, respectively (Fig.
5A). We found that the addition of CX3CR1-ODNs led to a progressive decrease in the contents of TNF $\alpha$ and IL$1 \beta$ in the BV2 microglial culture medium compared with scrambled-ODNs, but CX3CL1-ODNs did not result in any significant change (Fig. 5B). These results indicate that CX3CR1 perhaps played a more important role for miR195-induced effects in the brains of the ischemic mice. 


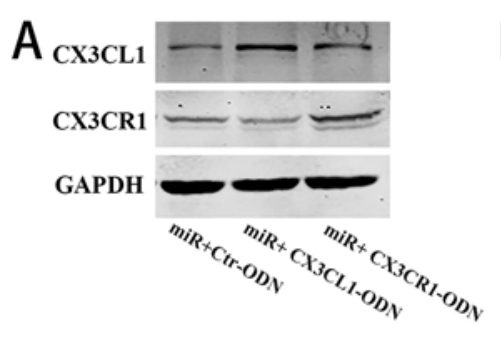

D

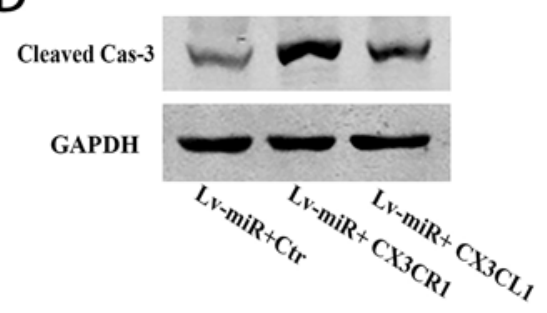

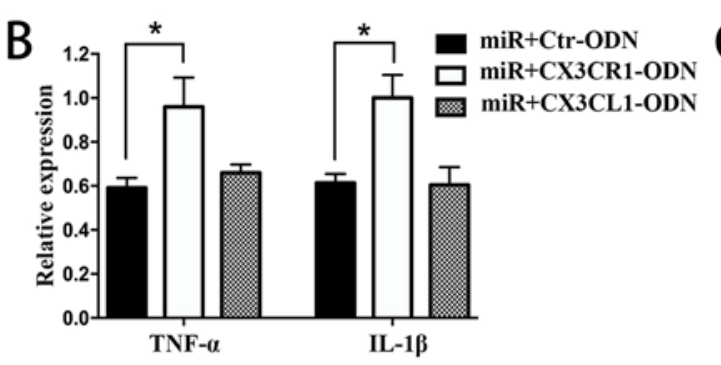

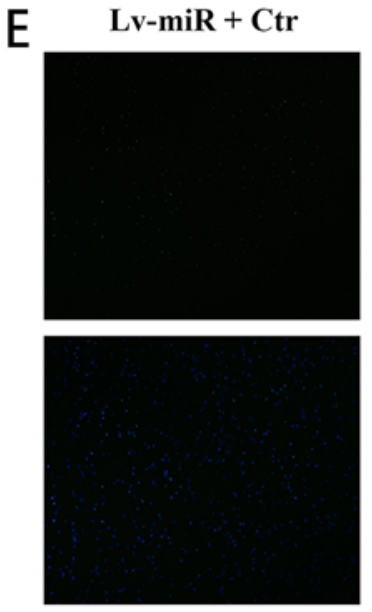

Lv-miR + CX3CR1
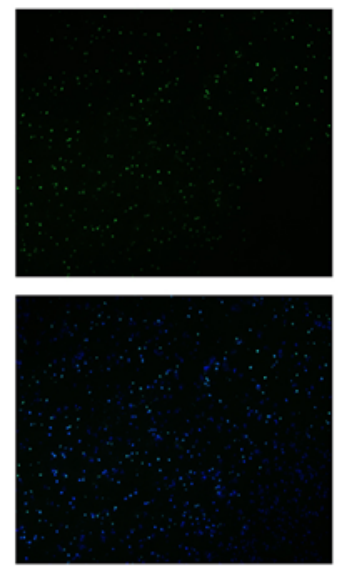

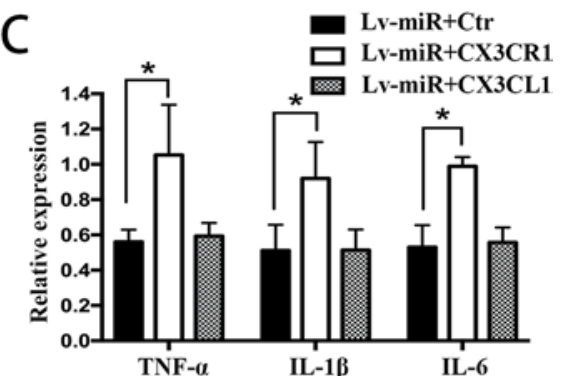

Lv-miR + CX3RL1
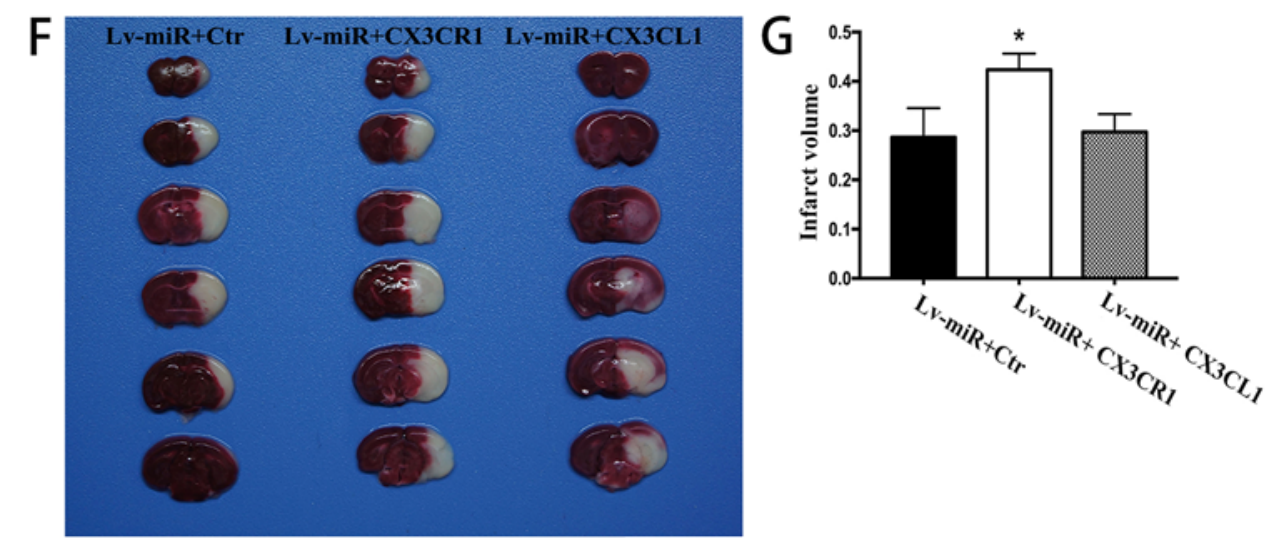

FIG. 5. Neuroprotective effect of miR-195 relies mainly on inhibiting CX3CL1. A: CX3CL1 and CX3CR1 protein expression was determined by Western blotting in the BV2 cells cotransfected with miR-195 mimics and scrambled control-ODN, CX3CL1-ODN, or CX3CR1-ODN. B: The expression of TNF $\alpha$ and IL-1 $\beta$ was detected using ELISA in the OGD-treated BV2 cells cotransfected with miR-195 mimics and scrambled control-ODN, CX3CL1-ODN, or CX3CR1-ODN. C: The expression of TNF $\alpha$, IL-1 $\beta$, and IL-6 was detected using ELISA in the periinfarct areas of mouse brains coinfected with Lv-miR-195 and Lv-control, Lv-CX3CL1, or LV-CX3CR1. D: The caspase-3 protein was determined by Western blotting in the periinfarct areas of the mouse brains coinfected with Lv-miR-195 and Lv-control, Lv-CX3CL1, or Lv-CX3CR1. E: The apoptotic cells are shown by TUNEL in the periinfarct areas of a mouse brain coinfected with Lv-miR-195 and Lv-Ctr, Lv-CX3CL1, or Lv-CX3CR1. F: Cerebral infarct volume was evaluated by TTC staining of coronal sections of mouse brains coinfected with Lv-miR-195 and LV-control, Lv-CX3CL1, or Lv-CX3CR1. Data are presented as the mean \pm SEM for 3 separate experiments performed in duplicate. ${ }^{*} p<0.05$. Figure is available in color online only.

To further investigate the essential role of CX3CL1 and CX3CR1 in the miR-195-induced effects, we constructed and infused Lv-CX3CL1 or Lv-CX3CR1 into Lv-miR-195infected mice. Similarly to the cytological experiments, in these in vivo experiments the effects of miR-195 on the expression of TNF $\alpha$, IL-1 $\beta$, and IL-6 were significantly reversed in the periinfarct areas of mouse brain infected with Lv-CX3CR1, but there was no significant change in mice infected with Lv-CX3CL1 (Fig. 5C). Meanwhile, the neuroprotective effects regulated by ectopic expression of miR-195 were rescued by Lv-CX3CR1, but were less affected by Lv-CX3CL1 (Fig. 5D and E). Furthermore, mice coinfused with Lv-CX3CR1 and Lv-miR-195 had a significantly increased infarct volume (Fig. 5F). These data suggest that both CX3CL1 and CX3CR1 are direct targets of miR-195, but that inhibition of CX3CR1 is essential for 
miR-195-induced neuroprotection and suppression of inflammatory signaling.

\section{Discussion}

An unregulated inflammatory response by ischemiaactivated microglia is responsible for postischemic neuronal damage; however, the molecular mechanism has not yet been fully clarified. In the present study, we found that miR-195 was downregulated in a mouse model of cerebral ischemia and also in ischemic stroke patients, and we demonstrated that prevention of a decrease in miR-195 expression in the brain subsequent to focal cerebral ischemia may be neuroprotective. Furthermore, we confirmed that miR-195 exerts neuroprotective roles mainly through CX3CR1-mediated neuroinflammation, and then subsequently by inhibiting neuronal cell apoptosis, with both CX3CL1 and CX3CR1 as direct targets of miR-195.

Our study demonstrated the role of miR-195 mainly in cellular and animal experiments and confirmed the expression of miR-195 through detection in ischemic stroke patients. Therefore, the main limitation of this study is that we did not investigate the correlation between miR-195 expression in the blood of ischemic stroke patients and their clinical outcome, and that we cannot verify the protective effect of miR-195 in clinical practice, because the application of miRNA has not yet reached the clinical stage.

In our experiment, we found that the levels of miR-195 were significantly decreased in the plasma of ischemic stroke patients and in mice suffering experimental ischemic stroke, findings which suggest that miR-195 might be crucial for suppressing ischemic injury. Then we further demonstrated that miR-195 has a neuronal protection function in cerebral ischemia. In vivo, our findings demonstrate that intra-cerebroventricular infusion of miR-195 not only reduces brain infarct volumes but also improves the functional neurological deficits in mice with cerebral ischemia, highlighting a possible clinical role for miR-195 in acute stroke treatment. Furthermore, we also found that miR-195 could alleviate neuronal apoptosis induced by in vivo MCAO and in vitro OGD. Previous studies have reported that miR-195 exerts proapoptotic effects in many different tumor cells. ${ }^{8,26}$ However, in the present study, we observed that miR-195 showed antiapoptotic effects in neurons during cerebral ischemia, which is consistent with the findings of Ai et al. ${ }^{1,2,14} \mathrm{We}$ speculate that these seemingly conflicting results may be due to the protean effects of miRNAs on different cells and genes. These results require further investigation.

In this study, we also observed a novel mechanism by which miR-195 protects the brain against ischemic injury through inhibiting neuroinflammation and subsequent neuronal apoptosis. It is known that cerebral ischemia induces acute inflammation, which exacerbates brain injury. Gonsalves and Kalra reported that miR-195 targets macrophage inflammatory protein $-1 \beta$ (MIP-1 $\beta$ ), suggesting that miR-195 might be involved in the inflammatory response. ${ }^{6}$ Additionally, in silico analysis further showed potential binding sites for miR-195 on 3'UTRs of both CX3CL1 and CX3CR1. CX3CL1 is expressed predominantly by neurons, while CX3CR1 as the CX3CL1 receptor is highly ex- pressed within microglia. The CX3CL1/CX3CR1 signaling pathway functions between neurons and microglia and has been shown to play a key regulatory role in neuroinflammation. ${ }^{13,16}$ Therefore, we hypothesize that miR-195 may be involved in the neuroinflammatory response. In vivo, we found that miR-195 overexpression could inhibit the inflammatory response in MCAO mice, as shown by the suppression of the inflammatory cytokines TNF $\alpha$, IL-1 $\beta$, and IL-6. In addition, we consistently observed the same results in BV2 cells. We further identified an inverse relationship between miR-195 and the expression of CX3CL1 and CX$3 \mathrm{CR} 1$, and we verified that CX3CL1 and CX3CR1 are the direct targets of miR-195 using the dual luciferase reporter assay, while miR-195 exerts neuroprotective roles mainly through CX3CR1-mediated neuroinflammation and inhibition of subsequent neuronal cell apoptosis. Actually, whether CX3CL1/CX3CR1 signaling is neuroprotective or neurotoxic is quite debatable. For instance, Donohue et al. proved that higher plasma CX3CL1 is associated with better outcome from ischemic stroke, ${ }^{5}$ and Cipriani et al. demonstrated that acute administration of CX3CL1 reduces ischemic damage via an adenosine-dependent mechanism in permanent focal cerebral ischemia. ${ }^{3}$ However, new evidence has indicated that inhibition of CX3CL1/CX3CR1 signaling could reduce recruitment of peripheral macrophages, activation of microglia and macrophages, and expression of inflammatory cytokines, as well as protect neurological function. ${ }^{7,15,16,21}$ As such, the neuroprotective role of miR-195 through CX3CR1-mediated neuroinflammation certainly needs further study. Our previous research revealed that NF- $\kappa \mathrm{B}$ as a transcription factor can regulate the expression of miR-195, and NF- $\kappa \mathrm{B}$ has been reported to participate in the inflammatory process. ${ }^{1}$ The data we report here strongly support our hypothesis that miR-195 is also involved in the neuroinflammatory response. However, in complicated inflammatory networks that occur in conditions such as cerebral ischemia, further investigation of the function and mechanism of miR-195 is still needed.

\section{Conclusions}

We discovered that miR-195 promotes neuronal cell survival against cerebral ischemic damage by inhibiting CX3CR1-mediated neuroinflammation in a mouse model, and we confirmed that miR-195 was significantly downregulated in a large cohort $(n=96)$ of ischemic stroke patients in a single institution. This study explored in detail the neuroprotective mechanism of miR-195 and for the first time verified the antiinflammatory effects of miR-195.

\section{Acknowledgments}

This work was supported by the National Natural Science Foundations of China (81572482, 81602225, 81302178, 81500912); the Natural Science Foundations of Heilongjiang Province of China (QC2013C096); and The Project of Science and Technology of Education Department of Heilongjiang Province (12541308).

\section{References}

1. Ai J, Sun LH, Che H, Zhang R, Zhang TZ, Wu WC, et al: MicroRNA-195 protects against dementia induced by chronic 
brain hypoperfusion via its anti-amyloidogenic effect in rats. J Neurosci 33:3989-4001, 2013

2. Chen X, Jiang XM, Zhao LJ, Sun LL, Yan ML, Tian Y, et al: MicroRNA-195 prevents dendritic degeneration and neuron death in rats following chronic brain hypoperfusion. Cell Death Dis 8: 2850, 2017

3. Cipriani R, Villa P, Chece G, Lauro C, Paladini A, Micotti E, et al: CX3CL1 is neuroprotective in permanent focal cerebral ischemia in rodents. J Neurosci 31:16327-16335, 2011

4. Daou B, Kent AP, Montano M, Chalouhi N, Starke RM, Tjoumakaris S, et al: Decompressive hemicraniectomy: predictors of functional outcome in patients with ischemic stroke. J Neurosurg 124:1773-1779, 2016

5. Donohue MM, Cain K, Zierath D, Shibata D, Tanzi PM, Becker KJ: Higher plasma fractalkine is associated with better 6-month outcome from ischemic stroke. Stroke 43:23002306, 2012

6. Gonsalves C, Kalra VK: Endothelin-1-induced macrophage inflammatory protein- $1 \beta$ expression in monocytic cells involves hypoxia-inducible factor- $1 \alpha$ and AP-1 and is negatively regulated by microRNA-195. J Immunol 185:6253-6264, 2010

7. Grosse GM, Tryc AB, Dirks M, Schuppner R, Pflugrad H, Lichtinghagen $\mathrm{R}$, et al: The temporal dynamics of plasma fractalkine levels in ischemic stroke: association with clinical severity and outcome. J Neuroinflammation 11:74, 2014

8. Guo J, Wang M, Liu X: MicroRNA-195 suppresses tumor cell proliferation and metastasis by directly targeting BCOX1 in prostate carcinoma. J Exp Clin Cancer Res 34:91, 2015

9. Hentschel KA, Daou B, Chalouhi N, Starke RM, Clark S, Gandhe A, et al: Comparison of non-stent retriever and stent retriever mechanical thrombectomy devices for the endovascular treatment of acute ischemic stroke. J Neurosurg 126:1123-1130, 2017

10. Huang W, Liu X, Cao J, Meng F, Li M, Chen B, et al: miR134 regulates ischemia/reperfusion injury-induced neuronal cell death by regulating CREB signaling. J Mol Neurosci 55:821-829, 2016

11. Jickling GC, Ander BP, Shroff N, Orantia M, Stamova B, Dykstra-Aiello C, et al: Leukocyte response is regulated by microRNA let7i in patients with acute ischemic stroke. Neurology 87:2198-2205, 2016

12. Kernan WN, Ovbiagele B, Black HR, Bravata DM, Chimowitz MI, Ezekowitz MD, et al: Guidelines for the prevention of stroke in patients with stroke and transient ischemic attack: a guideline for healthcare professionals from the American Heart Association/American Stroke Association. Stroke 45:2160-2236, 2014 (Erratum in Stroke 46:e54, 2015)

13. Limatola C, Ransohoff RM: Modulating neurotoxicity through CX3CL1/CX3CR1 signaling. Front Cell Neurosci 8:229, 2014

14. Liu CD, Wang Q, Zong DK, Pei SC, Yan Y, Yan ML, et al: Knockdown of microRNA-195 contributes to protein phosphatase-2A inactivation in rats with chronic brain hypoperfusion. Neurobiol Aging 45:76-87, 2016

15. Liu Y, Wu XM, Luo QQ, Huang S, Yang QW, Wang FX, et al: CX3CL1/CX3CR1-mediated microglia activation plays a detrimental role in ischemic mice brain via p38MAPK/PKC pathway. J Cereb Blood Flow Metab 35:1623-1631, 2015

16. Liu YZ, Wang C, Wang Q, Lin YZ, Ge YS, Li DM, et al: Role of fractalkine/CX3CR1 signaling pathway in the recov- ery of neurological function after early ischemic stroke in a rat model. Life Sci 184:87-94, 2017

17. Selvaraj UM, Ortega SB, Hu R, Gilchrist R, Kong X, Partin A, et al: Preconditioning-induced CXCL12 upregulation minimizes leukocyte infiltration after stroke in ischemia-tolerant mice. J Cereb Blood Flow Metab 37:801-813, 2017

18. Shen MY, Chen FY, Hsu JF, Fu RH, Chang CM, Chang $\mathrm{CT}$, et al: Plasma L5 levels are elevated in ischemic stroke patients and enhance platelet aggregation. Blood 127:13361345,2016

19. Stary CM, Xu L, Sun X, Ouyang YB, White RE, Leong J, et al: MicroRNA-200c contributes to injury from transient focal cerebral ischemia by targeting Reelin. Stroke 46:551-556, 2015

20. Sun LH, Ban T, Liu CD, Chen QX, Wang X, Yan ML, et al: Activation of Cdk5/p25 and tau phosphorylation following chronic brain hypoperfusion in rats involves microRNA-195 down-regulation. J Neurochem 134:1139-1151, 2015

21. Tang Z, Gan Y, Liu Q, Yin JX, Liu Q, Shi J, et al: CX3CR1 deficiency suppresses activation and neurotoxicity of microglia/macrophage in experimental ischemic stroke. J Neuroinflammation 11:26, 2014

22. Tu WJ, Zeng XW, Deng A, Zhao SJ, Luo DZ, Ma GZ, et al: Circulating FABP4 (fatty acid-binding protein 4) is a novel prognostic biomarker in patients with acute ischemic stroke. Stroke 48:1531-1538, 2017

23. Turner RC, Dodson SC, Rosen CL, Huber JD: The science of cerebral ischemia and the quest for neuroprotection: navigating past failure to future success. J Neurosurg 118:10721085,2013

24. Wang Y, Chen H, Fu Y, Ai A, Xue S, Lyu Q, et al: MiR-195 inhibits proliferation and growth and induces apoptosis of endometrial stromal cells by targeting FKN. Int J Clin Exp Pathol 6:2824-2834, 2013

25. Yang X, Tang X, Sun P, Shi Y, Liu K, Hassan SH, et al: MicroRNA-15a/16-1 antagomir ameliorates ischemic brain injury in experimental stroke. Stroke 48:1941-1947, 2017

26. Zhao N, Li S, Wang R, Xiao M, Meng Y, Zeng C, et al: Expression of microRNA-195 is transactivated by Sp1 but inhibited by histone deacetylase 3 in hepatocellular carcinoma cells. Biochim Biophys Acta 1859:933-942, 2016

\section{Disclosures}

The authors report no conflict of interest concerning the materials or methods used in this study or the findings specified in this paper.

\section{Author Contributions}

Conception and design: Zhao, G Yang, Liu, L Wang, Chen. Acquisition of data: Liu, X Wang, Dong, Zhang, Z Yang, Sun, Xue, X Wang, Gao, Li, Ilgiz. Analysis and interpretation of data: G Yang, L Wang. Drafting the article: G Yang, L Wang. Critically revising the article: Chen, Sun, Yi. Approved the final version of the manuscript on behalf of all authors: Zhao. Statistical analysis: Zhou. Study supervision: Zhao, Li, Ai.

\section{Correspondence}

Shiguang Zhao: The First Affiliated Hospital of Harbin Medical University, Heilongjiang Province, China.guangsz@hotmail.com. 